\title{
SLOWLY VARYING FUNCTIONS IN THE COMPLEX PLANE
}

\author{
BY \\ MONIQUE VUILLEUMIER
}

\begin{abstract}
Let $f$ be analytic and have no zeros in $\mid$ arg $z \mid<\alpha<\pi$; $f$ is called slowly varying if, for every $\lambda>0, f(\lambda z) / f(z) \rightarrow 1$ uniformly in |arg $z \mid$ $<\beta<\alpha$, when $|z| \rightarrow \infty$. One shows that $f$ is slowly varying if and only if $z f^{\prime}(z) / f(z) \rightarrow 0$ uniformly in $\operatorname{larg} z \mid<\beta<\alpha$, when $|z| \rightarrow \infty$.
\end{abstract}

1. Introduction. In 1930, Karamata [1] introduced a class of functions called slowly varying, which have since been applied in various fields of mathematics. Precisely, a real valued function $f$, positive and measurable on the positive real axis, is called slowly varying if $f(\lambda x) / f(x) \rightarrow 1(x \rightarrow \infty)$ for all $\lambda>0$.

Typical elementary functions belonging to this class are the iterated log functions, $\log t, \log \log t, \ldots$ Clearly these functions are restriction to the real axis of analytic functions, $\log z, \log \log z, \ldots$, which have the asymptotic property

$$
f(\lambda z) / f(z) \rightarrow 1 \text { when } z \rightarrow \infty,
$$

for any complex $\lambda \neq 0$. It seems therefore natural to investigate analytic functions satisfying condition (1.1), or some similar condition. Such an investigation has not yet been done; although the concept of slow variation has been extended to functions of several variables (see [2]), an extension to analytic functions of a complex variable is only suggested in [3].

In $\S 2$, we shall give a definition of analytic functions slowly varying as $z \rightarrow \infty$ in a sector $\operatorname{larg} z \mid<\alpha \leqslant \pi$ and establish some fundamental properties of these functions. The most significant one is expressed by property (2.2) in Theorem 1, or equivalently by Theorem 1". The definition given here is only one of two possible equally natural definitions; however, it follows from Theorem 1 that these two possible definitions are really equivalent (see paragraph following Theorem $1 ")$. Also in $\S 2$, we shall define analytic regularly varying functions.

In $\S 3$, we present an application of the preceding results to a problem in the theory of entire functions. In connection with the principle of PhragmenLindelöf for entire functions of finite order, M. L. Cartwright [4] introduces some comparison functions. It is easy to see that these comparison functions are parti-

Received by the editors March 21, 1975.

AMS (MOS) subject classificattons (1970). Primary 30A84.

Key words and phrases. Slowly varying functions. 
cular cases of analytic regularly varying functions. The remarks that constitute $\$ 3$ will show that the conditions that define the comparison functions are redundant and can be simplified.

2. Main results. The functions considered in this section will be defined on a sector $S_{R}(\alpha)=\{z:|z|>R,|\arg z|<\alpha\}$ where $R>0$ and $0<\alpha \leqslant \pi$. $H_{R}(\alpha)=\{z: \operatorname{Re} z>\log R,|\operatorname{Im} z|<\alpha\}$ where $R>0$ and $0<\alpha \leqslant \pi$.

For the sake of simplicity, we shall say that $f(z) \rightarrow A$ almost uniformly in $S_{R}(\alpha)$ when $|z| \rightarrow \infty$ (or in $H_{R}(\alpha)$ when $\operatorname{Re} z \rightarrow \infty$ ) if the convergence is uniform in $\arg z$ for $\operatorname{larg} z \mid \leqslant \beta$, for any $\beta \in(0, \alpha)$ (or uniform in $\operatorname{Im} z$ for $|\operatorname{Im} z|$ $\leqslant \beta$, for any $\beta \in(0, \alpha))$.

Definition. A function $f$ is called slowly varying in $S_{R}(\alpha)$ if $f$ is analytic and has no zeros in $S_{R}(\alpha)$ and if for every $\lambda>0, f(\lambda z) / f(z) \rightarrow 1$ almost uniformly in $S_{R}(\alpha)$ when $|z| \rightarrow \infty$.

The fundamental property of slowly varying functions is given by the following theorem.

THEOREM 1. Let $f$ be analytic and have no zeros in the sector $S_{R}(\alpha)$. Then, the following two statements are equivalent:

(2.1) for any $\lambda>0, f(\lambda z) / f(z) \rightarrow 1$ almost uniformly in $S_{R}(\alpha)$ when $|z| \rightarrow \infty$;

(2.2) $z f^{\prime}(z) / f(z) \rightarrow 0$ almost uniformly in $S_{R}(\alpha)$ when $|z| \rightarrow \infty$.

Since the analytic function $f$ has no zeros in the simply-connected domain $S_{R}(\alpha)$, there exists a function $F$, analytic in $H_{R}(\alpha)$ and such that

$$
e^{F(u)}=f\left(e^{u}\right) \quad \text { for every } u \in H_{R}(\alpha) .
$$

From (2.3), it follows immediately that Theorem 1 is equivalent to the following one.

THEOREM 1'. Let $F$ be analytic in the half-strip $H_{R}(\alpha)$. Then, the following two statements are equivalent:

(2.4) for every real $t, F(z+t)-F(z) \rightarrow 0$ almost uniformly in $H_{R}(\alpha)$ when $\operatorname{Re} z \rightarrow \infty$;

(2.5) $F^{\prime}(z) \rightarrow 0$ almost uniformly in $H_{R}(\alpha)$ when $\operatorname{Re} z \rightarrow \infty$.

Proof of Theorem $1^{\prime}$. Implication $(2.5) \Rightarrow(2.4)$ is obvious since $F(z+t)-F(z)=\int_{z}^{z+t} F^{\prime}(u) d u$.

To prove that (2.4) implies (2.5), we first show that $F(u+t)-F(u)$ is uniformly bounded for $t \in[0,1], \operatorname{Re} u \geqslant x$ and $|\operatorname{Im} u| \leqslant \gamma$, whenever $\gamma \in(0$, $\alpha)$. To this end, let us define $K(z)=\{t: t \in[0,1],|F(z+t)-F(z)| \leqslant 1\}$, for every $z \in H_{R}(\alpha)$. Let then $K_{n}$ denote the intersection of all sets $K(z)$ for $\operatorname{Re} z>n$ and $|\operatorname{Im} z| \leqslant \gamma$. Since $K(z)$ is closed, the sets $K_{n}$ are also closed, and 
because of (2.4), $\bigcup_{n=1}^{\infty} K_{n}=[0,1]$. It follows from Baire's category theorem that one of the sets $K_{n}$ must contain a nondegenerate closed interval. In other words, there exists a subinterval $[a, b]$ of $[0,1]$ and a positive integer $N_{0}$ such that

$$
|F(z+t)-F(z)| \leqslant 1 \quad \text { whenever } t \in[a, b], \operatorname{Re} z \geqslant N_{0},|\operatorname{Im} z| \leqslant \gamma .
$$

It follows that

$$
|F(z+t)-F(z+a)| \leqslant 2 \text { if } t \in[a, b], \operatorname{Re} z \geqslant N_{0},|\operatorname{Im} z| \leqslant \gamma,
$$

which can be rewritten as

$$
|F(z+t)-F(z)| \leqslant 2 \quad \text { if } t \in[0, b-a], \operatorname{Re} z \geqslant N_{1},|\operatorname{Im} z| \leqslant \gamma .
$$

This last inequality implies that

$$
|F(z+t)-F(z)| \leqslant 2 k \quad \text { if } t \in[0, k(b-a)], \operatorname{Re} z \geqslant N_{1},|\operatorname{Im} z| \leqslant \gamma .
$$

In particular, there exist $M$ and $N$ such that

$$
|F(z+t)-F(z)| \leqslant M \text { if } t \in[0,1], \operatorname{Re} z \geqslant N,|\operatorname{Im} z| \leqslant \gamma .
$$

Now, let us write $F(z)=G(z)+H(z)$, with

$$
G(z)=\int_{0}^{1}[F(z)-F(z+t)] d t \text { and } H(z)=\int_{0}^{1} F(z+t) d t=\int_{z}^{z+1} F(u) d u \text {. }
$$

Clearly, by (2.4), $H^{\prime}(z) \rightarrow 0$ almost uniformly in $H_{R}(\alpha)$ when $\operatorname{Re} z \rightarrow \infty$. We shall now show that (2.6) implies that $G^{\prime}(z) \rightarrow 0$ almost uniformly in $H_{R}(\alpha)$ when $\operatorname{Re} z \rightarrow \infty$. This will complete the proof of (2.5).

For every $\beta, 0<\beta<\alpha$, let $\gamma \in(\beta, \alpha)$. Let $x=\operatorname{Re} z$ and let

$$
\Phi(x, t)=\operatorname{Max}_{|\operatorname{Im} z|<\gamma}|F(z)-F(z+t)| .
$$

Then, by (2.4), $\Phi(x, t) \rightarrow 0$ as $x \rightarrow \infty$, for any real $t$, and by $(2.6), \Phi(x, t) \leqslant$ $M$ for all $t \in[0,1]$ and $x \geqslant N$. So, from the definition of $G(z)$ and from Lebesgue's bounded convergence theorem, we obtain

i.e.

$$
\underset{|\operatorname{Im} z|<\gamma}{\operatorname{Max}}|G(z)| \leqslant \int_{0}^{1} \Phi(x, t) d t \rightarrow 0 \quad(x \rightarrow \infty),
$$

(2.7) $G(z) \rightarrow 0$ uniformly in $\operatorname{Im} z$, for $|\operatorname{Im} z| \leqslant \gamma$, when $\operatorname{Re} z \rightarrow \infty$.

It remains now to show that (2.7) implies that

(2.8) $G^{\prime}(z) \rightarrow 0$ uniformly in $\operatorname{Im} z$, for $|\operatorname{Im} z| \leqslant \beta$, when $\operatorname{Re} z \rightarrow 0$.

Let $|\operatorname{Im} z| \leqslant \beta$ and let $\rho=\gamma-\beta$. By Cauchy's integral formula, we have 


$$
G^{\prime}(z)=\frac{1}{2 \pi i} \int_{|u-z|=\rho} \frac{G(u)}{(u-z)^{2}} d u,
$$

so that $\left|G^{\prime}(z)\right| \leqslant 1 / p \operatorname{Max}_{|u-z|=\rho}|G(u)|$, which implies that

$$
\left|G^{\prime}(z)\right| \leqslant \frac{1}{\rho} \sup _{|\operatorname{Im} u|<\gamma, \operatorname{Re} u \geqslant x-\rho} G(u),
$$

for all $z$ such that $|\operatorname{Im} z| \leqslant \beta, \operatorname{Re} z \geqslant x$. Now (2.8) follows from this last inequality and (2.7).

The fundamental theorem for slowly varying functions of a real variable is Karamata's representation theorem: $f$ is slowly varying if and only if $f(x)=$ $c(x) \exp \left(\int_{x_{0}}^{x} \delta(t) / t d t\right)$, where $c(x) \rightarrow c$ as $x \rightarrow+\infty, c>0$, and $\delta(x)$ is a continuous function tending to 0 as $x \rightarrow \infty$ (see [1]).

If $f$ is a function analytic and with no zeros in $S_{R}(\alpha)$, let $\epsilon(z)=$ $z f^{\prime}(z) / f(z)$, or equivalently $f(z)=C \exp \left(\int_{z_{0}}^{z} \epsilon(u) / u d u\right)$, where $C$ is a constant different from 0 . Then Theorem 1 can be reformulated as the analogue of Karamata's representation theorem for analytic slowly varying functions. It will read as follows.

THEOREM 1". A function $f$, analytic and with no zeros in $S_{R}(\alpha)$, is slowly varying in $S_{R}(\alpha)$ if and only if $f$ can be represented in the form

$$
f(z)=C \exp \left(\int_{z_{0}}^{z} \frac{\epsilon(u)}{u} d u\right)
$$

where $C$ is a constant different from 0 , and $\epsilon(z)$ is a function analytic in $S_{R}(\alpha)$ and tending to 0 almost uniformly in $S_{R}(\alpha)$ when $|z| \rightarrow \infty$.

In defining analytic slowly varying functions we have required that $f(\lambda z) / f(z)$ tends to 1 only for real positive values of $\lambda$. Another definition seems also natural. It would require that the same condition be fulfilled for $\lambda$ complex, suitably restricted so that $\lambda z$ remain in the domain of definition of the function $f$. However, the two definitions are equivalent. Indeed, it is easy to deduce from Theorem 1 " the following corollary, which shows that the limit property $f(\lambda z) / f(z) \rightarrow 1$ is true for $\lambda$ complex in $S_{R}(\alpha)$, and moreover, uniformly in $\lambda$ in a compact subset of $S_{R}(\alpha)$.

COROLlaRY. Suppose $f$ is slowly varying in $S_{R}(\alpha)$, i.e. $f$ is analytic, has no zeros in $S_{R}(\alpha)$ and satisfies condition (2.1). Let $S=\left\{z: R_{1} \leqslant|z| \leqslant R_{2}\right.$, $\operatorname{larg} z \mid \leqslant \beta\} \subset S_{R}(\alpha)$, and let $\gamma \in(0, \alpha-\beta)$. Then $f(\lambda z) / f(z) \rightarrow 1(|z| \rightarrow \infty)$ uniformly in $\lambda \in S$ and $\operatorname{larg} z \mid \leqslant \gamma$.

Real slowly varying functions $L(t)$ are often used in expressions of the form $f(t)=t^{\alpha} L(t)$. Clearly, such a function has the property that $f(\lambda t) / f(t) \rightarrow \lambda^{\alpha}$ 
$(t \rightarrow \infty)$. In fact, these functions, called regularly varying, are characterized by a simpler limit property, namely that $\lim _{t \rightarrow \infty} f(\lambda t) / f(t)$ exists and is different from 0 (see [1]).

In a similar way, a complex valued regularly varying function will be defined as a function $f$, analytic and without zeros in $S_{R}(\alpha)$, which satisfies $\lim _{|z| \rightarrow \infty} f(\lambda z) / f(z)=h(\lambda) \neq 0$, almost uniformly in $S_{R}(\alpha)$ for all $\lambda$ real and positive.

Clearly, $h$ satisfies the functional equation $h(\lambda \mu)=h(\lambda) h(\mu)$. Consequently, $h(\lambda)$ must be of the form $h(\lambda)=\lambda^{\alpha}$, where $\alpha$ is complex. Putting $f(z)=z^{\alpha} L(z)$ ( $z^{\alpha}$ means its principal value), it follows that $\lim _{|z| \rightarrow \infty} L(\lambda z) / L(z)=1$ almost uniformly in $S_{R}(\alpha)$, i.e. that $L(z)$ is slowly varying.

3. Application. In the study of complex functions which are analytic in a sector $\alpha \leqslant \arg z \leqslant \beta, z \geqslant R$, and which are of finite order $\rho^{\prime}$, an important role is played by a function $h(\theta)$ which is defined by

$$
h(\theta)=\limsup _{r \rightarrow \infty} \frac{\log \left|f\left(r e^{i \theta}\right)\right|}{r^{0}}
$$

where $0<\rho<\rho^{\prime}$. In $[4$, p. 41], M. L. Cartwright defines a more general function $h(\theta)$, by replacing the comparison function $r^{0}$ by a function $V$ which has the following properties:

(i) $V(z)$ is analytic in the sector considered,

(ii) $V(x)>0$ and $V(x)$ is increasing for $x>x_{0}$,

(iii) $\lim \sup _{r \rightarrow \infty} \log V(r) / \log r=\rho$,

(iv) $\lim _{r \rightarrow \infty} V\left(r e^{i \theta}\right) / V(r)=e^{i \rho \theta}$ uniformly in $\theta$ in the sector considered,

(v) $\lim _{r \rightarrow \infty} V(k r) / V(r)=k^{\rho}$ for every $k>1$.

We shall show that condition (iii) is redundant and that conditions (iv) and (v) are equivalent to the condition

(3.1) $V(z)=z^{\rho} L(z), L$ slowly varying as $z \rightarrow \infty$ in $\{z:|z| \geqslant R$, $\alpha \leqslant \arg z \leqslant \beta\}$.

From the definition of slowly varying functions, respectively from the corollary in $\S 2$, we obtain that (3.1) implies (v), resp. (iv). On the other hand, since condition (v) is obviously satisfied for all real positive $k$, writing

$$
\frac{V(\lambda z)}{V(z)}=\frac{V(\lambda z)}{V(\lambda|z|)} \frac{V(\lambda|z|)}{V(|z|)} \frac{V(|z|)}{V(z)}
$$

we deduce from (iv) and (v) that

$$
V(\lambda z) / V(z) \rightarrow \lambda^{\rho} \text { uniformly in arg } z \in(\alpha, \beta) \text { as }|z| \rightarrow \infty,
$$

for any $\lambda$ positive. By Theorem $1^{\prime \prime}$, it follows then from (3.1) that 


$$
\frac{\log V(r)}{\log r}=\rho+\frac{1}{\log r}\left\{C+\int_{1}^{r} \frac{\epsilon(t)}{t} d t\right\}
$$

where $\epsilon(t) \rightarrow 0(t \rightarrow \infty)$. As a result

(iii) $\lim _{r \rightarrow \infty} \log V(r) / \log r=\rho$.

Thus, not only (iii), but even (iii)' is a consequence of (iv) and (v).

\section{REFERENCES}

1. J. Karamata, Sur un mode de croissance régulière des fonctions, Mathematica (Cluj) 4 (1930), 38-53.

2. B. Bajšanski and J. Karamata, Regularly varying functions and the principle of equicontinuity, Publ. Ramanujan Institute no. 1 (1968/69), 235-246. MR 42 \#3222.

3. J. Karamata, Some theorems concerning slowly varying functions, Math. Res. Center, U.S. Army, Tech. Sum. Report No. 432, Madison, Wisconsin, 1963.

4. M. L. Cartwright, Integral functions, Cambridge Tracts in Math. and Math. Phys., no. 44, Cambridge Univ. Press, Cambridge, 1956. MR 17, 1067.

DEPARTMENT OF MATHEMATICS, OHIO STATE UNIVERSITY, COLUMBUS, 